\title{
Genotype x Environment Interaction and Stability Analysis for Earliness, Seed Yield and Fruit Yield in Okra Using the Additive Main Effect and Multiplicative Interaction (AMMI)
}

\author{
Sanket J. More $^{1 *}$, K.N. Chaudhari ${ }^{2}$, G.B. Vaidya ${ }^{3}$ and S.L. Chawla ${ }^{2}$ \\ ${ }^{1}$ ICAR - Central Tuber Crops Research Institute, Sreekariyam PO, Thiruvananthapuram, \\ Kerala-695 017, India \\ ${ }^{2}$ ASPEE College of Horticulture and Forestry, Navsari Agricultural University, \\ Navsari-396 450, India \\ ${ }^{3}$ ANKUR seed co. PVT. LTD., Nagpur, India \\ *Corresponding author
}

\section{A B S T R A C T}

Fifty five okra genotypes (ten lines, four testers, forty hybrids and one check) okra accessions were tested for stability in three different environments between 2014-15 using

Keywords

Genotype x Environment Interaction, Stability

Analysis, Additive Main Effect and Multiplicative Interaction (AMMI),

Abelmoschus esculentus (L.) Moench

\section{Article Info}

Accepted: 04 February 2018 Available Online: 10 March 2018 Additive main effect and multiplicative interaction (AMMI) and Genotype main effect and Genotype by Environment (GGE) models. The experiment was laid out in a Randomized Complete Block Design (RCBD) with three replications. The mean squares due to genotypes were highly significant for all the traits when tested against pooled error and pooled deviation which indicated the presence of considerable genetic variability in the materials. Highly significant differences were also observed amongst environments for all the traits when tested against pooled error and pooled deviation which indicated the presence of considerable environmental differences for all the traits. The genotypes $\mathrm{x}$ environment interactions were significant for all the traits except fruit length when tested against pooled error. Both AMMI and GGE biplots identified most stable, high yielding genotypes that were overall best in performance in relation to yield and stability. Result of stability estimates of 55 genotypes revealed that none of the genotype was stable for all the traits studied. Further, IC - 045796 x GAO - 5 and VIO 47672 x GAO - 5 were the most stable hybrids in terms of fruit yield and its components. For the trait, fruit yield per plant Environment $\mathrm{E}_{3}$ (winter season) was found to be the most unfavourable while Environment $\mathrm{E}_{2}$ (rainy season) was the most favourable environment.

\section{Introduction}

Okra [Abelmoschus esculentus (L.) Moench] belongs to the cotton family Malvaceae. This warm season crop considered to have originated from India. Okra is economically and traditionally important vegetable of tropical and sub-tropical countries of the world such as India, West Africa, South East Asia, Southern America, Brazil, Turkey and northern Australia (Rao, 1985). In India, it is grown during summer as well as in rainy season. According to the $2^{\text {nd }}$ Advance Estimate of NHB Database (2017), India is 
going to produce 6.1 million tons of okra from an area of 0.5 million hectare with the productivity of 12 tonnes/ha, making India the largest producer and consumer of okra in the world. India is a major okra producing country in the world comprising of $71 \%$ of total area under okra (FAOSTAT, 2014). Several high yielding hybrids have been developed by Indian Institute of Vegetable Research (ICARIIVR), Varanasi, India among which "Kashi Bhairav" has achieved yield up to 20-22 MT/ha (Anonymous, 2016). As mentioned earlier, an average productivity of okra in India is 12.00 tonnes/ha, a large potential yield gap exists between farmers' yield and than that of documented by various research organizations. The major problem in okra cultivation is lack of high-yielding varieties/hybrids. The productivity of okra should be increased through hybridization and recombination followed by stability analysis (Solankey et al., 2016). Several researchers have studied stability analysis in okra in details. They have also reported stable okra hybrids by using the Additive Main Effect and Multiplicative Interaction.

In multi-environment trails genotype $\mathrm{X}$ environment interactions is often exist (Simmonds, 1991; Kang, 1998). If there were no interaction, there is no need to conduct yield trails over different locations/environments, as trial may be conducted at only one location and providing universal results (Gauch and Zobel, 1996). Such an ideal situation does not exist in reality. Genotype x environment interaction is expected in plant breeding experiments that involve cultivars of diverse genetic background and diverse test sites (Kang et al., 2006).

Genotype $\mathrm{x}$ environment interaction study is important to breeders to develop not only improved but also stable varieties, suitable for a particular location or multi-location. The goal of any plant breeding programme is to develop cultivars with high yielding potential with stable performance over a wide range of environments (Olayiwola and Ariyo, 2013). Performance of various okra genotypes keeps varying in varying environmental conditions. The genotypic and environmental interactions are usually present under all conditions in pure lines, hybrids, synthetics or any other material used for breeding which complicate the breeding work and forbid the progress of the crop improvement programmes. Thus, it is imperative to study the performance of a crop in more than one environment to identify genotypes, which give high stability for various yield related traits over a wide range of environment (Jindal et al., 2008). The phenomenon of genotype - environment interaction is a common problem in plant breeding program and has long been a challenge to plant breeder. A variety developed by a plant breeder is usually grown at different locations for many years under different conditions. Assessing any genotype without including its interaction is incomplete and thus limits the accuracy of yield estimates. It is usually preferable to estimate yield stability and reliability values with reference to all GE interaction effects (Ezekiel et al., 2011).

The objective of this research is evaluate the efficiency of the combined use of AMMI and GGE technique to study GEI of various traits of fifty five okra genotypes in three different seasons.

\section{Materials and Methods}

The study was conducted during September, 2013 to February, 2015 (including crossing programme during September to December, 2013). The experiment (55 genotypes including 14 parents, their 40 hybrids and one standard check) was laid out in a randomized complete block design (RBD) with three 
replications over three environments (Table 1) at the Regional Horticultural Research Station, ASPEE College of Horticulture and Forestry, Navsari Agricultural University, Navsari, situated at coastal region of South Gujarat. Geographically, it is situated at $20^{\circ}-37^{\prime} \mathrm{N}$ latitude and $72^{\circ}$ - 54' E longitude with an altitude of 11.98 meters above the Mean Sea Level. All recommended horticultural practices along with plant protection measures were followed uniformly and as and when required.

\section{Parental material and recorded data}

Fourteen diverse parents (Table 2) were crossed in line $\mathrm{x}$ tester fashion to produce forty $F_{1}$ hybrids. Selfed parental seeds, hybrid seeds and standard check were treated with Thiram $4 \mathrm{~g} / \mathrm{kg}$ of seeds were sown (10 plants/row) in RBD fashion at spacing of $60 \mathrm{x}$ $45 \mathrm{~cm}$. Randomly five plants from each plot were selected to record the observation on days to first flowering, first flowering node, plant height, number of branches, number of fruits per plant, fruit yield per plant, fruit length, fruit weight, number of seeds per pod and 100 seed weight. Plot wise means for various traits were subjected to Analysis of Variance (ANOVA) and means were separated using the Duncan Multiple range test. Further, data were analyzed statistically for stability parameters based on mean performance across the seasons as per the model suggested by Eberhart and Rusell (1966) for various traits. Data was analysed using Windostat Version 8.6 (Indostat services).

\section{Results and Discussion}

Analysis of variance for phenotypic
stability

Stability performance is one of the most desirable properties of a genotype for its wide adaptation. The mean squares for phenotypic stability for different traits are presented in Table 3. The mean squares due to genotypes were highly significant for all the traits when tested against pooled error and pooled deviation which indicated the presence of considerable genetic variability in the material tested. Highly significant differences were also observed amongst environments for all the traits when tested against pooled error and pooled deviation which indicated the presence of considerable environmental differences for all the traits. The genotypes $\mathrm{x}$ environment interactions were significant for all the traits except fruit length when tested against pooled error. This indicated that genotype interacted significantly in different environments. The mean square due to genotype $\mathrm{x}$ environment interactions were also significant for days to first flowering, number of fruits per plant, plant height, fruit yield per plant and fruit weight when tested against pooled deviation. The lack of significant $\mathrm{G} \times \mathrm{E}$ interaction for rest of the traits under study indicated that genotypes responded consistently over the environments for these traits, hence, the results of these traits are not included in the study. The significance of $\mathrm{G}$ x $\mathrm{E}$ interactions have also been reported by Srivastava et al., (2011), Ezekiel et al., (2011), Alake and Ariyo (2012), Hamed and Hafiz (2012), Olayiwola and Ariyo (2013) and Javia (2014).

The mean squares due to environments (linear) were highly significant for all the traits when tested against pooled deviation except for leaves per plant. However, the same was significant for all the traits when tested against pooled error. This indicated that variation among environments was linear and it signifies unit change in environmental index for each unit change in the environmental conditions. The variance due to $\mathrm{G} \times \mathrm{E}$ were further partitioned in to components (i) $\mathrm{G} \times \mathrm{E}$ (linear) and (ii) $\mathrm{G} \times \mathrm{E}$ (non-linear) i.e. pooled deviation. $\mathrm{G} \times \mathrm{E}$ (linear) was found to be 
significant for all the traits except number of branches per plant and fruit length when tested against pooled error indicating differential performance of genotypes under diverse environments but with considerably varying norms, i.e., the linear sensitivity of different genotypes is variable.

\section{Stability estimates}

Stability estimated to assess the stability over the environments is presented in Table 4. For days to first flowering, a perusal of the data revealed that non-linear component was significant for 3 genotypes, indicating larger contribution of non-linear component towards $\mathrm{G} \times \mathrm{E}$ interaction. Among parents, line, IC 111493 and tester, Arka Anamika were found stable as they were flowered earlier and exhibited unit regression coefficient along with non-significant value of deviation from regression. Looking to the data of plant height, significant linear and non-linear components were reflected by 2 and 10 genotypes respectively, thereby suggesting major role of non-linear component towards $G \quad x \quad E$ interaction.

Seven hybrids recorded dwarfism (lower mean values for plant height) with non-significant regression coefficient and least deviation from linear regression thus, identified as average stable for this trait. The best three among them were EC - 284327 x GJO - $3(128.16 \mathrm{~cm})$, JOL - 08 - 13 x GAO - $5(131.47 \mathrm{~cm})$, EC 284327 x GAO - 5 (134.50 cm), JOL - 08 13 x Varsha Uphar $(135.38 \mathrm{~cm})$ and EC 305623 x Arka Anamika $(135.50 \mathrm{~cm})$. Among hybrids, IC - 045796 x GAO - 5 (249.26 g) had high mean fruit yield per plant with regression coefficient near unity and nonsignificant deviation from regression. It also manifested average stability for days to first flowering and number of fruits per plant, followed by cross VIO 47672 x GAO - 5 (241.57 g), manifested average stability for days to first flowering and fruit weight. In general, the hybrid which found stable for fruit yield also depicted stability in respect of its one or more yield component (Table 4). This indicated that the stability of various component traits might be responsible for observed stability of hybrids for fruit yield. The chance for selection of stable genotypes could be strengthened by selection in favour of stability in some yield component. Grafius (1956) also suggested that the stability of fruit yield might be due to the stability of various yield components. Phenotypic stability of various component traits reflecting into fruit yield stability were also reported by various workers viz., Kachhadia et al., (2011), Akotkar et al., (2011), Srivastava et al., (2011) and Javia (2014) in okra.

Varietal improvement can encouraged by accumulation of favourable genes for yield and tolerance to various stresses (Singh, 1993). It will not be wrong to say that the accrual of all high yielding genes into one cultivar is nearly impossible task. Genotype $\mathrm{x}$ environment interaction plays an important role in the overall performance of a cultivar, so it becomes utmost important to identify high yielding stable varieties across multiple environments or a cultivar that have specific adaptation in specific environment/location.

Multi-location or multi-environment screening of genotypes offers opportunities for selecting genetically adapted and specifically adapted cultivars. 40 hybrids were developed by using 10 lines and 4 testers and were evaluated along with parents and one commercial check in three different environments. Obtained data was subjected to AMMI and GGE biplot analysis. GGE biplot identified cultivar. Figure 1 represents the biplot of AMMI results. The $y-$ axis represents the IPCA 1 scores, while the $\mathrm{x}$ axis represents the main effects of days to first flowering (A), first flowering node (B) and number of seeds $(C)$, respectively. 
Fig.1 Additive main effect and multiplicative interaction (AMMI) and Genotype $\times$ environment interaction (GGE) biplot analysis of (A) days to first flowering, (B) first flowering node and (C) number of seeds for 55 genotypes of okra established in 3 environments
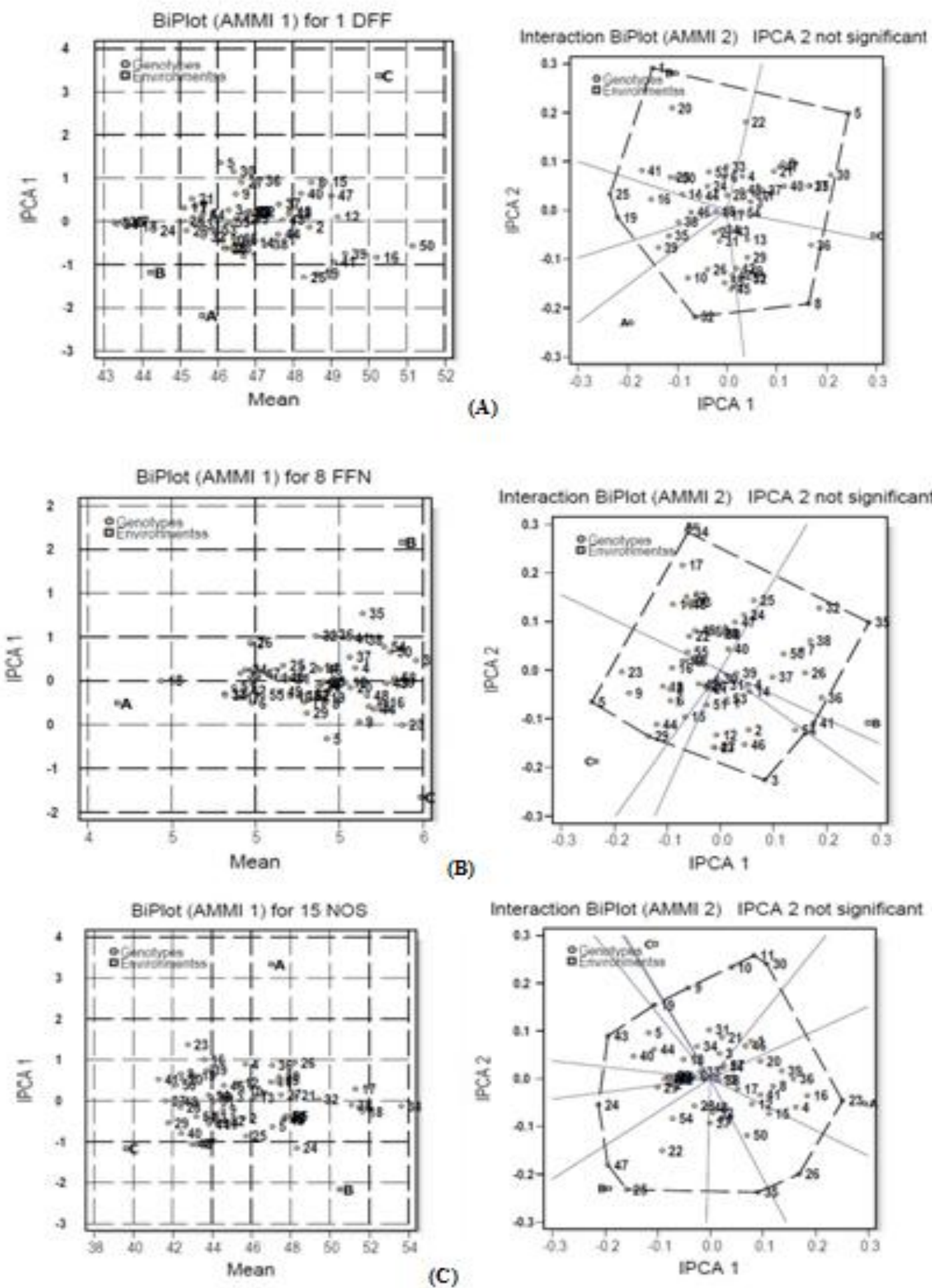

(B)

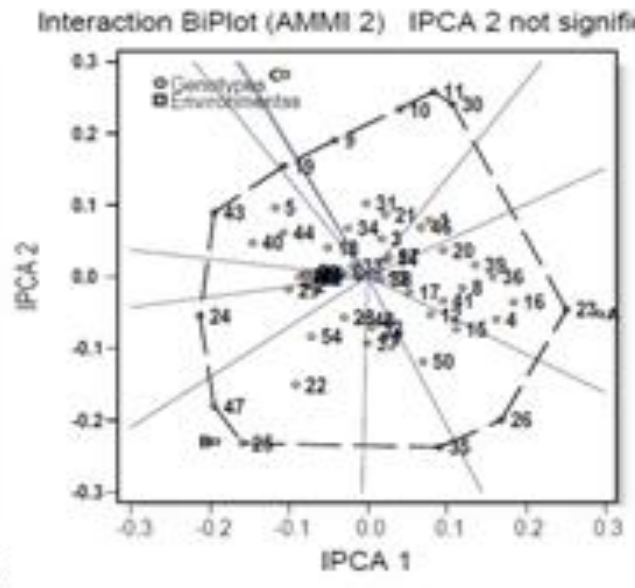


Table.3 Analysis of variance for phenotypic stability pertaining to various traits

\begin{tabular}{|c|c|c|c|c|c|c|}
\hline \multirow[t]{2}{*}{ Source } & \multirow[t]{2}{*}{ df } & & & & & \\
\hline & & Days to first flowering & First flowering node & Plant height $(\mathrm{cm})$ & Number of branches per plant & Number of fruits per plant \\
\hline Genotype (G) & 54 & $8.723^{* *++}$ & 0.326 & $225.387 * *++$ & $0.088^{* *++}$ & $7.101 * *++$ \\
\hline Planting date $(\mathbf{P})$ & 2 & $549.338 * *++$ & $56.936^{* *++}$ & $198051.08 * *++$ & $0.190 * *++$ & $1909.41 * *++$ \\
\hline $\mathbf{G} \times \mathbf{P}$ & 108 & $3.769 * *+$ & $0.316^{* *}$ & $100.430^{* *++}$ & $0.014 * *$ & $2.799 * *++$ \\
\hline Planting date(linear) & 1 & $1098.676 * *++$ & $113.872 * *++$ & $396102.17 * *++$ & $0.381 * *++$ & $3818.831 * *++$ \\
\hline $\mathbf{G} \times \mathbf{E}$ (linear) & 54 & $5.114 * *++$ & $0.288^{* *}$ & $126.194 * *+$ & 0.011 & $4.001 * *++$ \\
\hline Pooled deviation & 55 & $2.38 *$ & $0.34 * *$ & $73.31^{* *}$ & $1.75 * *$ & $1.57 * *$ \\
\hline Pooled error & 324 & 1.652 & 0.0593 & 33.145 & 0.010 & 0.711 \\
\hline \multirow[t]{2}{*}{ Source } & \multirow[t]{2}{*}{ df } & & & & & \\
\hline & & Fruit yield per plant (g) & Fruit length $(\mathrm{cm})$ & Fruit weight (g) & Number of seed per fruit & 100 seed weight $(\mathrm{g})$ \\
\hline Genotype (G) & 54 & $2000.29 * *++$ & $0.695^{* *++}$ & $0.6805^{* *++}$ & $25.120 * *++$ & $0.2039 * *++$ \\
\hline Planting date $(\mathbf{P})$ & 2 & $465953.706^{* *++}$ & $266.64 * *++$ & $58.005 * *++$ & $1704.88 * *++$ & $7.940 * *++$ \\
\hline $\mathbf{G} \times \mathbf{P}$ & 108 & $617.68 * *++$ & 0.2244 & $0.246 * *++$ & $5.03 * *$ & $0.0643 * *$ \\
\hline Planting date(linear) & 1 & $931907.41 * *++$ & $533.29 * *++$ & $116.01 * *++$ & $3409.702 * *++$ & $15.88^{* *++}$ \\
\hline $\mathbf{G} \times \mathbf{P}$ (linear) & 54 & $905.400 * *++$ & 0.1746 & $0.327^{* *++}+$ & $4.637 * *$ & $0.066^{*}$ \\
\hline Pooled deviation & 55 & $323.97 * *$ & 0.27 & 0.16 & $5.33 * *$ & 0.06 \\
\hline Pooled error & 324 & 79.735 & 0.190 & 0.167 & 2.923 & 0.045 \\
\hline
\end{tabular}

*, ** significant at 5 and $1 \%$ level, respectively against pooled error.

,+++ Significant at 5 and $1 \%$ level, respectively against pooled deviation.

Table.4 Stability parameters of individual genotypes for days to first flowering, plant height (cm), number of fruit per plant, fruit yield per plant $(\mathrm{g})$ and fruit weight $(\mathrm{g})$

\begin{tabular}{|c|c|c|c|c|c|c|c|c|c|c|c|c|c|c|c|c|}
\hline \multirow{2}{*}{$\begin{array}{l}\text { Sr. } \\
\text { no. }\end{array}$} & \multirow[t]{2}{*}{ Genotype } & \multicolumn{3}{|c|}{ Days to first flowering } & \multicolumn{3}{|c|}{ Plant height (cm) } & \multicolumn{3}{|c|}{ Number of fruit per plant } & \multicolumn{3}{|c|}{ Fruit yield per plant (g) } & \multicolumn{3}{|c|}{ Fruit weight (g) } \\
\hline & & Mean & $\mathrm{b}_{\mathrm{i}}$ & $\mathrm{S}^{2} \mathrm{~d}_{\mathrm{i}}$ & Mean & $b_{i}$ & $\mathrm{~S}^{2} \mathrm{~d}_{\mathrm{i}}$ & Mean & $\mathrm{b}_{\mathrm{i}}$ & $\mathrm{S}^{2} \mathrm{~d}_{\mathrm{i}}$ & Mean & $b_{i}$ & $\mathrm{~S}^{2} \mathrm{~d}_{\mathrm{i}}$ & Mean & $b_{i}$ & $\mathrm{~S}^{2} \mathrm{~d}_{\mathrm{i}}$ \\
\hline \multicolumn{17}{|c|}{ Female parent (Lines) } \\
\hline 1. & VIO 44244 & 49.08 & 0.28 & 4.57 & 123.37 & 0.99 & 15.03 & 14.59 & 0.87 & 0.85 & 163.75 & $0.786^{*}$ & -78.305 & 10.28 & 0.512 & 0.469 \\
\hline 2. & IC -111493 & 46.48 & 0.90 & 1.32 & 134.97 & 0.83 & 34.89 & 17.25 & 1.15 & -0.69 & 207.31 & 1.103 & -58.726 & 10.89 & 1.103 & -0.165 \\
\hline 3. & JOL $-08-13$ & 47.72 & 0.98 & -1.23 & 126.51 & 1.06 & -21.20 & 15.76 & 0.91 & 0.09 & 179.59 & 0.935 & 141.460 & 10.55 & 1.142 & -0.103 \\
\hline 4. & EC - 284327 & 47.60 & 0.77 & -0.99 & 124.55 & 0.94 & -11.62 & 16.57 & 1.07 & 1.33 & 190.35 & 1.044 & $337.167 *$ & 10.62 & 0.864 & -0.103 \\
\hline 5. & IC - 045796 & 46.78 & 0.85 & 2.57 & 135.20 & 0.83 & 51.48 & 17.28 & 1.15 & -0.69 & 206.12 & 1.130 & -62.633 & 10.88 & 1.111 & -0.163 \\
\hline 6. & IC -052273 & 46.48 & 0.65 & -1.31 & 132.50 & 1.00 & -30.93 & 15.20 & 0.61 & 0.57 & 175.44 & 0.676 & 212.717 & 10.58 & 0.471 & -0.116 \\
\hline 7. & $\mathrm{JOL}-10-18$ & 49.01 & 1.60 & -1.62 & 131.47 & 0.84 & $101.09 *$ & 15.18 & 0.75 & -0.26 & 175.55 & 0.745 & -63.646 & 10.61 & 0.522 & -0.132 \\
\hline 8. & $\mathrm{AOL}-09-17$ & 47.89 & 1.19 & -1.59 & 127.60 & 1.12 & 10.70 & 15.65 & 1.07 & 0.90 & 177.44 & 0.950 & -45.985 & 10.30 & 0.935 & 0.140 \\
\hline 9. & VIO 47672 & 46.74 & 1.02 & 1.64 & 135.47 & 0.84 & 49.53 & 17.26 & 1.15 & -0.69 & 204.27 & 1.132 & -60.460 & 10.86 & 1.113 & -0.166 \\
\hline 10. & $\mathrm{EC}-305623$ & 51.16 & 0.59 & 1.24 & 126.43 & 0.91 & $127.92 *$ & 14.66 & 0.74 & 1.88 & 161.89 & 0.718 & -39.982 & 10.36 & $0.447^{*}$ & -0.166 \\
\hline \multicolumn{17}{|c|}{ Male parent (Tester) } \\
\hline 11. & GAO -5 & 46.85 & 1.03 & 2.50 & 134.47 & 0.82 & 63.61 & 17.24 & 1.15 & -0.71 & 204.98 & 1.144 & -39.399 & 10.85 & 1.102 & -0.164 \\
\hline
\end{tabular}




\begin{tabular}{|c|c|c|c|c|c|c|c|c|c|c|c|c|c|c|c|c|}
\hline 12. & $\mathrm{GJO}-3$ & 46.95 & 1.03 & 2.60 & 134.03 & 0.80 & 59.60 & 17.27 & 1.15 & -0.69 & 203.54 & 1.132 & -51.231 & 10.86 & 1.062 & -0.162 \\
\hline 13. & Arka Anamika & 45.93 & 0.91 & -0.02 & 120.91 & 1.02 & 43.82 & 14.81 & $0.72 *$ & 2.61 & 166.54 & 0.760 & 121.643 & 10.65 & 1.384 & -0.144 \\
\hline 14. & Varsha Uphar & 45.61 & 1.13 & -1.55 & 130.04 & 1.01 & 86.86 & 14.36 & $0.50 * *$ & 13.78 & 164.24 & 0.527 & $2409.921 * *$ & 10.69 & 1.050 & -0.110 \\
\hline \multicolumn{17}{|c|}{ Hybrid } \\
\hline 15. & VIO $44244 \times$ GAO -5 & 46.70 & 0.61 & $22.15 * *$ & 130.93 & 1.07 & $144.97 *$ & 14.69 & 0.68 & $4.33 * *$ & 168.18 & 0.722 & $602.955 * *$ & 10.78 & 1.229 & -0.097 \\
\hline 16. & VIO $44244 \times$ GJO - 3 & 48.44 & 0.82 & -1.53 & 139.29 & 0.96 & 2.88 & 14.60 & $0.79 * *$ & -0.71 & 173.99 & 0.856 & -75.696 & 10.85 & 1.234 & -0.129 \\
\hline 17. & VIO $44244 \times$ ArkaAnamika & 46.30 & 1.24 & -1.60 & 137.96 & 0.94 & -1.39 & 14.23 & 0.81 & -0.07 & 169.63 & 0.858 & 25.952 & 10.74 & 1.650 & 0.208 \\
\hline 18. & VIO $44244 \times$ Varsha Uphar & 45.62 & 1.22 & -1.31 & 140.33 & 1.05 & -28.23 & 16.19 & $0.90^{* *}$ & -0.71 & 189.10 & 0.912 & -67.719 & 10.75 & 0.971 & -0.017 \\
\hline 19. & $\mathrm{IC}-111493 \times \mathrm{GAO}-5$ & 46.08 & 2.39 & -1.57 & 139.20 & 1.28 & $231.09 * *$ & 16.50 & 1.36 & $2.64 *$ & 204.41 & 1.421 & $1729.844 * *$ & 11.03 & 2.036 & -0.162 \\
\hline 20. & $\mathrm{IC}-111493 \times \mathrm{GJO}-3$ & 43.29 & 1.05 & -0.90 & 156.92 & 1.11 & -1.44 & 19.93 & 1.26 & 1.59 & 254.99 & 1.251 & $709.392 * *$ & 11.99 & 0.331 & $0.740^{*}$ \\
\hline 21. & IC $-111493 \times$ ArkaAnamika & 45.40 & 1.33 & -1.60 & 141.27 & 0.916 & -31.94 & 16.31 & 1.18 & -0.57 & 195.54 & 1.167 & 37.748 & 11.10 & 1.642 & 0.035 \\
\hline 22. & IC $-111493 \times$ Varsha Uphar & 48.48 & 1.56 & $12.67 * *$ & 137.12 & 1.14 & 86.57 & 16.27 & 0.89 & 0.52 & 185.30 & 0.956 & 150.140 & 10.44 & 0.707 & -0.166 \\
\hline 23. & $\mathrm{JOL}-08-13 \times \mathrm{GAO}-5$ & 46.49 & 1.64 & -1.61 & 131.47 & 0.98 & 30.71 & 17.19 & 1.25 & -0.67 & 199.95 & 1.196 & -58.718 & 10.83 & 1.376 & -0.099 \\
\hline 24. & $\mathrm{JOL}-08-13 \times \mathrm{GJO}-3$ & 46.15 & 0.46 & -0.63 & 128.44 & 1.15 & -21.86 & 16.58 & 1.02 & -0.71 & 193.96 & 1.070 & -79.727 & 10.69 & 0.811 & -0.142 \\
\hline 25. & JOL $-08-13 \times$ ArkaAnamika & 45.13 & 1.30 & -1.63 & 142.56 & 1.20 & 51.85 & 18.20 & 1.25 & -0.06 & 217.07 & 1.250 & -71.632 & 11.00 & 1.015 & -0.069 \\
\hline 26. & JOL $-08-13 \times$ Varsha Uphar & 49.18 & 0.95 & 0.99 & 135.38 & 0.94 & -1.35 & 17.40 & 1.22 & -0.59 & 207.54 & 1.230 & 38.424 & 10.95 & 1.257 & -0.161 \\
\hline 27. & $\mathrm{EC}-284327 \times \mathrm{GAO}-5$ & 47.92 & 1.12 & -0.44 & 134.50 & 0.97 & -29.32 & 15.77 & 1.04 & 0.00 & 185.02 & 1.021 & -17.663 & 10.84 & 1.481 & 0.096 \\
\hline 28. & $\mathrm{EC}-284327 \times \mathrm{GJO}-3$ & 46.92 & 0.61 & -0.25 & 128.16 & 1.08 & 35.01 & 15.31 & 0.66 & 0.79 & 173.14 & 0.732 & $258.483 *$ & 10.59 & 0.732 & -0.104 \\
\hline 29. & EC $-284327 \times$ ArkaAnamika & 48.85 & 1.85 & -0.92 & 127.05 & $0.7 *$ & -32.77 & 15.72 & $0.90 *$ & 2.23 & 174.33 & 0.766 & $847.149 * *$ & 10.27 & 1.040 & 0.105 \\
\hline 30. & EC $-284327 \times$ Varsha Uphar & 50.21 & 0.30 & 0.93 & 141.64 & 0.91 & -29.49 & 14.33 & 0.88 & 0.66 & 164.75 & 0.938 & 56.166 & 10.40 & 1.349 & -0.027 \\
\hline 31. & $\mathrm{IC}-045796 \times \mathrm{GAO}-5$ & 43.61 & 0.97 & -1.64 & 153.38 & 1.05 & -30.53 & 19.16 & 1.24 & -0.71 & 249.26 & 1.214 & -70.159 & 11.80 & $0.692^{*}$ & -0.166 \\
\hline 32. & IC $-045796 \times$ GJO -3 & 43.84 & 0.89 & -1.62 & 157.56 & 1.14 & 1.01 & 19.93 & 1.30 & 1.70 & 256.16 & 1.349 & $1092.592 * *$ & 11.87 & 0.518 & $1.073 * *$ \\
\hline 33. & IC $-045796 \times$ ArkaAnamika & 48.63 & -0.03 & 1.38 & 145.40 & 1.10 & 17.27 & 15.57 & 0.78 & -0.07 & 182.67 & 0.938 & 28.837 & 10.60 & 1.071 & -0.096 \\
\hline 34. & IC $-045796 \times$ Varsha Uphar & 46.18 & 0.73 & $10.82 * *$ & 142.62 & 1.04 & -29.51 & 14.37 & 0.60 & -0.62 & 163.73 & 0.711 & -52.109 & 10.64 & 0.789 & -0.160 \\
\hline 35. & IC $-052273 \times$ GAO -5 & 45.33 & 1.54 & -1.63 & 144.16 & 1.14 & -3.05 & 17.89 & 1.26 & 0.49 & 214.21 & 1.296 & $332.140 *$ & 10.96 & 1.078 & -0.026 \\
\hline 36. & $\mathrm{IC}-052273 \times \mathrm{GJO}-3$ & 46.79 & 1.38 & 1.88 & 130.10 & $0.7 *$ & -32.05 & 14.48 & $0.53 *$ & 3.16 & 165.31 & 0.520 & $709.693 * *$ & 10.79 & 0.729 & -0.147 \\
\hline 37. & IC $-052273 \times$ ArkaAnamika & 46.32 & 0.53 & 1.66 & 136.61 & 0.97 & 8.94 & 15.46 & 1.12 & -0.27 & 175.00 & 1.083 & -48.281 & 10.56 & 1.841 & -0.051 \\
\hline 38. & IC $-052273 \times$ Varsha Uphar & 44.31 & 0.87 & -0.70 & 145.46 & 1.13 & $354.2 * *$ & 17.88 & 1.20 ** & 4.31 & 219.95 & 1.233 & $1497.782 * *$ & 11.27 & 0.756 & 0.373 \\
\hline 39. & $\mathrm{JOL}-10-18 \times \mathrm{GAO}-5$ & 48.28 & -0.083 & 4.46 & 139.80 & 0.89 & 31.05 & 15.44 & 0.70 & -0.47 & 179.61 & 0.675 & -20.297 & 10.96 & 0.343 & -0.041 \\
\hline 40. & $\mathrm{JOL}-10-18 \times \mathrm{GJO}-3$ & 45.18 & 0.67 & -0.35 & 137.82 & 0.73 & -8.89 & 16.72 & 0.83 & 0.71 & 201.89 & 0.837 & $229.022 *$ & 11.15 & 0.740 & -0.039 \\
\hline 41. & JOL $-10-18 \times$ ArkaAnamika & 46.63 & 1.84 & -0.94 & 139.29 & 0.98 & -7.42 & 15.56 & 0.88 & 0.97 & 179.30 & 0.929 & -50.466 & 10.77 & 1.027 & -0.162 \\
\hline 42. & JOL $-10-18 \times$ Varsha Uphar & 45.08 & 1.03 & -1.51 & 143.75 & 1.07 & 34.94 & 16.84 & 1.21 & -0.51 & 192.45 & 1.158 & -33.689 & 10.48 & 1.543 & -0.012 \\
\hline 43. & $\mathrm{AOL}-09-17 \times \mathrm{GAO}-5$ & 46.85 & 1.08 & 0.74 & 141.84 & 1.01 & -16.03 & 16.65 & 1.23 & -0.53 & 189.89 & 1.163 & -42.677 & 10.41 & 1.636 & 0.119 \\
\hline 44. & $\mathrm{AOL}-09-17 \times \mathrm{GJO}-3$ & 46.42 & 2.07 & -0.75 & 146.30 & 1.24 & 16.65 & 18.00 & 1.36 & -0.13 & 216.23 & 1.309 & -57.347 & 11.06 & 1.401 & -0.154 \\
\hline 45. & AOL $-09-17 \times$ ArkaAnamika & 45.53 & 0.85 & -1.23 & 130.19 & 1.08 & $165.72 *$ & 15.90 & 1.06 & -0.35 & 182.95 & 1.031 & 10.252 & 10.53 & 1.256 & -0.165 \\
\hline 46. & AOL $-09-17 \times$ Varsha Uphar & 45.63 & 0.44 & 2.79 & 148.15 & 1.00 & -26.34 & 16.81 & 0.81 & 1.93 & 202.42 & 0.804 & 414.997* & 11.25 & 0.582 & -0.123 \\
\hline 47. & $\mathrm{VIO} 47672 \times \mathrm{GAO}-5$ & 43.49 & 1.09 & -0.44 & 154.60 & 1.11 & -16.64 & 18.92 & 1.27 & -0.01 & 241.57 & 1.367 & 215.430 & 11.65 & 0.868 & 0.095 \\
\hline 48. & VIO $47672 \times$ GJO - 3 & 43.34 & 0.88 & -1.51 & 160.70 & 1.15 & 2.19 & 20.92 & 1.37 & 1.52 & 278.01 & 1.452 & $611.988 * *$ & 12.26 & 0.298 & $0.522 *$ \\
\hline 49. & VIO $47672 \times$ ArkaAnamika & 46.20 & 0.38 & -1.50 & 141.90 & 0.98 & $148.76^{*}$ & 16.92 & 0.95 & -0.36 & 198.09 & 0.891 & 86.962 & 11.02 & 0.424 & 0.295 \\
\hline 50. & VIO $47672 \times$ Varsha Uphar & 47.09 & 1.73 & 3.92 & 133.51 & 0.73 & $112.36^{*}$ & 17.53 & 1.16 & -0.28 & 203.98 & 1.090 & 187.768 & 10.82 & 0.759 & -0.051 \\
\hline 51. & $\mathrm{EC}-305623 \times \mathrm{GAO}-5$ & 47.63 & 1.38 & -1.63 & 135.58 & 1.11 & $366.98^{* *}$ & 15.53 & $1.16^{*}$ & 8.44 & 178.67 & 1.161 & $1131.122^{* *}$ & 10.59 & 1.148 & 0.105 \\
\hline 52. & $\mathrm{EC}-305623 \times \mathrm{GJO}-3$ & 47.28 & 0.51 & -1.34 & 136.93 & 0.98 & $121.91 *$ & 14.81 & 0.63 & 1.55 & 167.15 & 0.668 & $473.822 * *$ & 10.57 & 0.707 & -0.137 \\
\hline 53. & EC $-305623 \times$ ArkaAnamika & 49.37 & 0.26 & -1.57 & 135.50 & 0.98 & 2.16 & 15.29 & 0.75 & -0.43 & 172.14 & $0.769^{*}$ & -77.946 & 10.54 & 0.867 & 0.024 \\
\hline 54. & EC - $305623 \times$ Varsha Uphar & 48.21 & 1.60 & -1.47 & 135.30 & 1.12 & -33.08 & 16.42 & 1.13 & 0.70 & 192.34 & 1.109 & 118.272 & 10.63 & 1.132 & -0.165 \\
\hline 55. & Check (Sonakshi) & 46.29 & 0.81 & 1.52 & 135.86 & 0.83 & 42.17 & 17.30 & 1.15 & -0.69 & 208.55 & 1.118 & -62.252 & 10.91 & 1.112 & -0.165 \\
\hline & Mean & 46.71 & 1.00 & - & 137.49 & 1.00 & - & 16.41 & 1.00 & - & 193.63 & 1.00 & - & 10.84 & 1.00 & - \\
\hline & SEM \pm & 1.09 & 0.34 & - & 12.7 & 0.1 & - & 6.1 & 0.01 & - & 0.10 & 0.33 & - & 0.07 & 1.36 & - \\
\hline
\end{tabular}




\section{Table.1 Details of seasons during the study}

Summer, 2014, February to May

Rainy, 2014, June to September

Winter, 2014-2015, November 2014 to February 2015

\begin{tabular}{|c|c|}
\hline Parent & Source/Origin \\
\hline VIO 44244 & AVRDC, Taiwan \\
\hline IC -111493 & NBPGR, New Delhi, India \\
\hline JOL $-08-13$ & Junagadh Agricultural University, Junagadh (Gujarat) \\
\hline $\mathrm{EC}-284327$ & NBPGR, New Delhi, India \\
\hline IC - 045796 & NBPGR, New Delhi, India \\
\hline IC -052273 & Junagadh Agricultural University, Junagadh (Gujarat) \\
\hline JOL $-10-18$ & Junagadh Agricultural University, Junagadh (Gujarat) \\
\hline $\mathrm{AOL}-09-17$ & Anand Agricultural University, Anand (Gujarat) \\
\hline VIO 47672 & AVRDC, Taiwan \\
\hline $\mathrm{EC}-305623$ & NBPGR, New Delhi, India \\
\hline \multicolumn{2}{|l|}{ Tester } \\
\hline GAO - 5 & Anand Agricultural University, Anand (Gujarat) \\
\hline GJO - 3 & Junagadh Agricultural University, Junagadh (Gujarat) \\
\hline ArkaAnamika & IIHR, Bengaluru, India \\
\hline Varsha Uphar & IIHR, Bengaluru, India \\
\hline
\end{tabular}

Table.5 Estimate of environmental index for various traits under different environments

\begin{tabular}{|c|l|c|c|c|}
\hline Sr. & Trait & \multicolumn{3}{|c|}{ Environmental index } \\
\cline { 2 - 4 } No. & & $\begin{array}{c}\mathrm{E}_{1} \\
(\text { Summer- } \\
\end{array}$ & $\begin{array}{c}\mathrm{E}_{2} \\
\text { (Rainy- }\end{array}$ & $\begin{array}{c}\mathrm{E}_{3} \\
\text { (Winter-2014- }\end{array}$ \\
\hline 1. & Days to first flowering & -1.099 & -2.646 & $15)$ \\
\hline 2. & $\begin{array}{l}\text { Number of branches per } \\
\text { plant }\end{array}$ & -0.062 & 0.007 & -0.005 \\
\hline 3. & Number of fruit per plant & 0.381 & 5.692 & -6.073 \\
\hline 4. & Plant height (cm) & -17.737 & 66.877 & -49.140 \\
\hline 5. & Fruit yield per plant (g) & -93.507 & 90.505 & 3.002 \\
\hline 6. & First flowering node & -1.173 & 0.528 & 0.645 \\
\hline 7. & Fruit length (cm) & 1.197 & 1.344 & -2.541 \\
\hline 8. & Fruit weight (g) & -0.120 & 1.081 & -0.692 \\
\hline 9. & Number of seed per fruit & 1.311 & 4.795 & -6.106 \\
\hline 10. & 100 seed weight (g) & 0.078 & 0.335 & -0.413 \\
\hline
\end{tabular}


Genotype VIO 47672 x GJO - 3 was the overall best of them all combing relative stability and registered less number of days to first flowering, followed by genotypes VIO 47672 x GAO - 5 and IC 045796 x GJO - 3. 20 genotypes registered early flowering. The poorest genotypes due to instability and late flowering were VIO47672 x Varsha Uphar and VIO 44244 x GJO - 3 (Fig. 1A). In case of first flowering node most of the hybrids were found highly stable and adapted to high performance environments. Genotype VIO $44244 \times$ Varsha Uphar was less than the average in terms of first flowering node. It was interesting to note that this particular genotype was highly stable in nature and was also adapted to low performance environment which was winter season in case of this study, depicting the scope of okra cultivation in off season (Fig. 1B). Lines, EC - 284327 and IC - 045796 were least stable among 55 genotypes and also registered late flowering. Figure 1C depicts the AMMI and GGebiplot analysis for number of seeds per fruit evaluated over three different environments. Most of the genotypes were found to be highly stable and adapted to high performance environment. Genotype IC - 045796 x Varsha Uphar was found to be highly stable in nature and adapted to high performance environment followed by VIO 44244 x Varsha Uphar. Genotype, JOL - 10 - 18 x Arka Anamika was found stable but recorded lowest mean for number of seeds per fruit (45.70). According to AMMI biplot analysis for number seeds per fruit, genotype, EC 284327 recorded less number of seeds than mean and were also highly instable. The biplot revealed the genotypes that performed best in each environment and the relationship between the environments. The relationship among environments was not so close. This is explained by larger angle between these environments, whereas there was a wider variation between the rainy season and the rest. The result of AMMI revealed that IC -
045796 x GAO - 5 and VIO 47672 x GAO 5 were the most stable genotypes because their interaction with the environment was not enough to hinder yield as indicated by their IPCA scores of zero and near.

Result of stability estimates of 55 genotypes revealed that none of the genotypes was stable for all the traits studied. Same results were reported by Patil et al., (2017). Thus, any generalization regarding stability of genotypes for all the traits is too difficult since the genotype may not simultaneously exhibit uniform responsiveness and stability patterns for all these traits. The yield is polygenically controlled complex trait and is being determined by the joint action of a number of component traits. Therefore, a proper understanding of relationship between fruit yield and its component traits could be of great help in choosing the proper components that may contribute not only towards the manifestation of complex trait but also towards its stability and association with high heterosis and desirable sca effects. The identification of parents having higher mean, good gca effects and high stability across the environments is of great value to the plant breeders while formulating breeding programme. Phenotypic stability of various component traits reflecting into fruit yield stability were also reported by various workers Akotkar et al., (2011), Srivastava et al., (2011) and Javia (2014) in okra.

\section{Environmental index}

The estimates of environmental indices revealed that the components traits for earliness and dwarfness viz, days to first flowering, plant height and first flowering node were favoured in $\mathrm{E}_{1}$ and $\mathrm{E}_{3}$, while the yield attributing traits like number of branches per plant, number of fruits per plant, fruit length and fruit weight were more favoured in $E_{2}$. For the trait fruit yield per 
plant $E_{3}$ was found to be the most unfavourable and $E_{2}$ the most favourable. In general, the environment $\mathrm{E}_{2}$ was found to be the most favourable for fruit yield and other related traits (Table 5).

\section{References}

Akotkar P.K., De D.K. and Ghoshdastidar K.K. 2011. Stability analysis for fruit yield and component characters in okra (Abelmoschus esculentus (L.) Moench). In: "National Seminar on Contemporary Approaches to Crop Improvement." Abstr., 62: 196.

Alake C.O. and Ariyo O.J. 2012. Comparative analysis of genotype $\mathrm{x}$ environment interaction in West African okra (Abelmoschus esculentus (L.) Moench). J. Agric. Sci. 4(4): 135 - 150.

Anonymous. 2016. Vegetable Varieties developed by IIVR. Retrieved from http://www.iivr.org.in/vegetablevarieties-developed-iivr

Eberhart S.A. and Russell W.A. 1966. Stability parameters for comparing varieties. Crop. Sci. 6: $26-40$.

Ezekiel S.A., Ariyo O.J. and Kehinde O.B. 2011. Stability assessment of some West African okra (Abelmoschus caillei) genotypes in Nigerian Genebank. J. Life Sciences 5: 906 912.

FAOSTAT. 2014. Production - Crops data. Food and Agriculture Organization of the United Nations. http://www.fao.org/ faostat.

Gauch H.G. Jr. and Zobel R.W. 1996. AMMI analysis of yield trials. In M.S. Kang and H.G. Gauch, Jr. (eds.), Genotypeby-Environment Interaction, pp. 85-122. CRC Press, Boca Raton, FL.

Grafius J. E. 1956. Components of yield in oats, a geometrical interpretation. Agron. J. 51: 515 - 554.

Hamed H. H. and Hafiz M.R. 2012. Selection of local okra (Abelmoschus esculentus
L.) genotypes for stability under saline conditions. Bulletin of Faculty of Agriculture, Cairo University, 63(2): $188-200$.

Javia R.M. 2014. Stability analysis for fruit yield and its attributing characters in okra [Abelmoschus esculentus (L.) Moench]. Int. J. Plant Sci. 9(1): 35 - 39.

Jindal S.K., Arora D. and Ghai T.R. 2008. Stability analysis for earliness in okra (Abelmoschus esculentus (L.) Moench), J. Res. Punjab Agric. Univ. 45(3 \& 4): $148-155$.

Kachhadia V. H., Dangaria C.J., Vachhani J.H., Jivani L.L. and Shekhat H.G. 2011. Satbility analysis in okra (Abelmoschus esculentus L. Moench). Int. J. Plant Sci. 6(1): 34 - 39.

Kang M.S. 1998. Using genotype-byenvironment interaction for crop cultivar development. Adv. Agron. 62:199-252.

Kang M.S., Aggarwal V.D. and Chirwa R.M. 2006. Adaptability and Stability of Bean Cultivars as Determined via YieldStability Statistic and GGE Biplot Analysis. J. Crop Improv. 15(1): 97120. doi: 10.1300/J411v15n01_08

NHB Database. 2017. 2nd Advance Estimate of Area and Production of Horticulture Crops (2016-2017). nhb.gov.in.

Olayiwola M. O. and Ariyo O.J. 2013. Relative discriminatory ability of GGE biplot and YSi in the analysis of genotype $\mathrm{x}$ Environment interaction in okra (Abelmoschus esculentus (L.) Moench). Int. J. Pl. Breed. and Genet. 7(3): $146-158$.

Patil S.S., Desai D.T., Patil P.P. and Sunayan R. 2017. Genotype X environment interaction for fruit yield and component characters in okra [Abelmoschus esculentus (L.) Moench]. Electron. J. Plant Breed. 8(3): 787-791.

Rao P.U. 1985. Chemical composition and biological evaluation of okra (Hibiscus 
esculentus) seeds and their kernels. Plant Foods Hum. Nutr. 35: 389-396.

Simmonds N.W. 1991. Selection for local adaptation in a plant breeding programme. Theor. Appl. Genet. 82:363-367.

Singh S.P. 1993. Bean genetics. In: A. van Schoonhoven and O. Voysest (eds.). Common Beans: Research for Crop Improvement, pp. 199-286. CABI Publishing, Wallingford, Oxon, UK.

Solankey S.S., Singh A.K. and Singh R.K. 2016. Heterosis of okra resistance sources for okra yellow vein mosaic virus (OYVMV) in okra (Abelmoschus esculentus). Indian J. Agr. Sci. 86: 1460-1465.

Srivastava K., Aparna J., Singh P.K. and Kumar S. 2011. Stability performance of okra hybrids over environments. In: "National Seminar on Contemporary Approaches to Crop Improvement." Abstr. 138: 244.

\section{How to cite this article:}

Sanket J. More, K.N. Chaudhari, G.B. Vaidya and Chawla, S.L. 2018. Genotype x Environment Interaction and Stability Analysis for Earliness, Seed Yield and Fruit Yield in Okra Using the Additive Main Effect and Multiplicative Interaction (AMMI). Int.J.Curr.Microbiol.App.Sci. 7(03): 373-383. doi: https://doi.org/10.20546/ijcmas.2018.703.043 